\title{
Application of Special FTIR ATR Techniques for Quantitative Structural Analysis of Thin Surface Layers
}

\author{
Dieter Baurecht, Gerald Reiter, Norbert Hassler, Michael Schwarzott, and Urs Peter Fringeli
}

\begin{abstract}
FTIR ATR spectroscopy is increasingly used for in situ investigations of processes at or near a surface. Particularly when thin layers (biomembranes, monolayers, thin films) are investigated with respect to surface concentration and molecular structure, very sensitive techniques have to be applied in order to achieve an adequate signal-to-noise ratio. This may lead to long measuring times due to extended data accumulation and averaging. However, this can cause new problems with respect to the stability of relevant experimental parameters, such as the sample itself, the spectrometer, and the atmosphere in the spectrometer.

In this article we report on two techniques which were developed or improved in our laboratory and successfully applied over past years. Both methods, the so-called single-beam sample reference (SBSR) spectroscopy and the modulation or modulated excitation (ME) spectroscopy, are well suited to compensate instabilities that occur in the course of an experimental series. The SBSR technique converts a single-beam FTIR spectrometer into a pseudo double-beam instrument. By this technique there is always a reference with the same age as the sample available. Moreover, by alternating sample and reference measurements within short time periods, varying environmental conditions such as water vapor concentration in the spectrometer are easily compensated. Moreover SBSR technique enables data evaluation in the conventional single-beam mode (SB) in both the sample (S) and reference (R) channel. This kind of evaluation is important to gain information on the history of $S$ and R. As examples for SBSR and SB applications we report on studies of the interaction of an endotoxin with an immobilized lipid bilayer membrane, as well as on the interaction of TNF $\alpha$ with a TNF $\alpha$ antibody. ME spectroscopy can be applied to systems that show a (pseudo-) reversible response to a periodic excitation. The response of the system measured with time-resolved FTIR spectroscopy is then processed by phase-sensitive detection (PSD). ME spectroscopy is able to determine kinetic constants of a system, allows a hardware separation of overlapping absorption bands, and eliminates all disturbing signal components which do not have the same frequency as the excitation itself. This improves the signal-to-noise ratio dramatically and leads in principal to a stable baseline. The binding of sodium cholate to an adsorbed protein layer of human serum albumin (HSA) is shown as an example that the required sensitivity to study specific molecular interaction is in the $\mu \mathrm{AU}$ range and can be reached by FTIR ME spectroscopy. In a second example, the measurement of structural changes of PLL induced by temperature modulation shows the feasibility of band separation and indicates the possible determination of kinetic properties of a system.
\end{abstract}

Keywords: Bio-interfaces · FTIR ATR · Modulated excitation · Specific recognition

\section{Introduction}

Knowledge of the molecular structure of thin surface layers is of essential interest for chemistry, biology, medicine, and pharmacy. Methods which are able to provide this information are e.g. X-ray photoelectron spectroscopy (XPS), time-of-flight secondary ion mass spectrometry (ToF-SIMS), infrared reflection absorption spectroscopy (IRRAS), and spectral ellipsometry (SE). Whereas XPS and ToF-SIMS belong to the group of destructive techniques, IRRAS and SE are examples for non-destructive techniques.

Among the latter methods, Fourier transform infrared attenuated total reflection (FTIR ATR) spectroscopy plays an important role. FTIR ATR techniques are used to qualitatively and quantitatively elucidate the physicochemical mechanisms of chemically, biologically or medically relevant surface processes on a molecular level. Whereas, e.g. XPS and ToF-SIMS provide insight into the distribution of elements and molecular structure of surface layers, FTIR ATR spectroscopy represents a sensitive method for in situ investigations of processes at or near a surface of interest (e.g. a model membrane, an immobilized protein, a polymer or a specifically modified organic or inorganic layer to be used as sensor). With the help of the measured spectra, information can be obtained about the functional groups of molecules, their quantity, and their spatial orientation. Even
E-Mail: urs.peter.fringeli@univie.ac.at

www.bpc.univie.ac.at 
additives and impurities of small amounts, which, however, may be able to strongly affect the surface properties, can be detected. Exact knowledge about a surface and its reactivity is a prerequisite for the useful application of surface modifying methods in order to obtain the information or product of interest.

Processes of adsorption and diffusion at polymer, biological or inorganic surfaces are of great medical and pharmaceutical importance (diagnostics, drug stability and application, biosensors) and play also an eminent role in inorganic and enzymatic catalysis. With FTIR ATR spectroscopy, such processes can be investigated in situ resulting in information on the surface concentration of an adsorbate, the conformation and orientation of functional groups, including protein backbone and side chains. Moreover, several time-resolved techniques enable access to dynamic processes. The special FTIR ATR techniques described in this article provide a better background compensation and an enhanced sensitivity compared to conventional single-beam measurements.

On the one hand, this is achieved by an optical attachment that converts a singlebeam FTIR spectrometer into a pseudo double-beam instrument. This technique is referred to as the single-beam-sample-reference (SBSR) method. On the other hand, modulated excitation (ME) spectroscopy is shown to result in utmost sensitivity and baseline stability combined with the ability to access the kinetics of a periodically stimulated process in the sample. The latter is the case if the period of stimulation is in the range of kinetic relaxation times of the excited process.

For a comprehensive summary of surface analytical methods in general and of the FTIR ATR techniques in particular see [1].

\section{Special FTIR ATR Techniques}

\subsection{SBSR ATR Spectroscopy}

Most FTIR spectrometers work in the single-beam (SB) mode. As a consequence a single channel reference (SC-R) spectrum has to be stored for later use to convert another single channel spectrum, e.g. that of the sample (SC-S) into a transmittance and/ or absorbance spectrum. This procedure favors inaccuracy due to any instabilities occurring between scanning SC-R and SC-S. Such drifts may result from the instrument itself or from the sample, as well as from disturbance by atmospheric absorptions, mainly from water vapor and carbon dioxide. In order to eliminate these unwanted effects to a great extent an ATR attachment has been constructed, converting a singlebeam instrument into a pseudo-double-

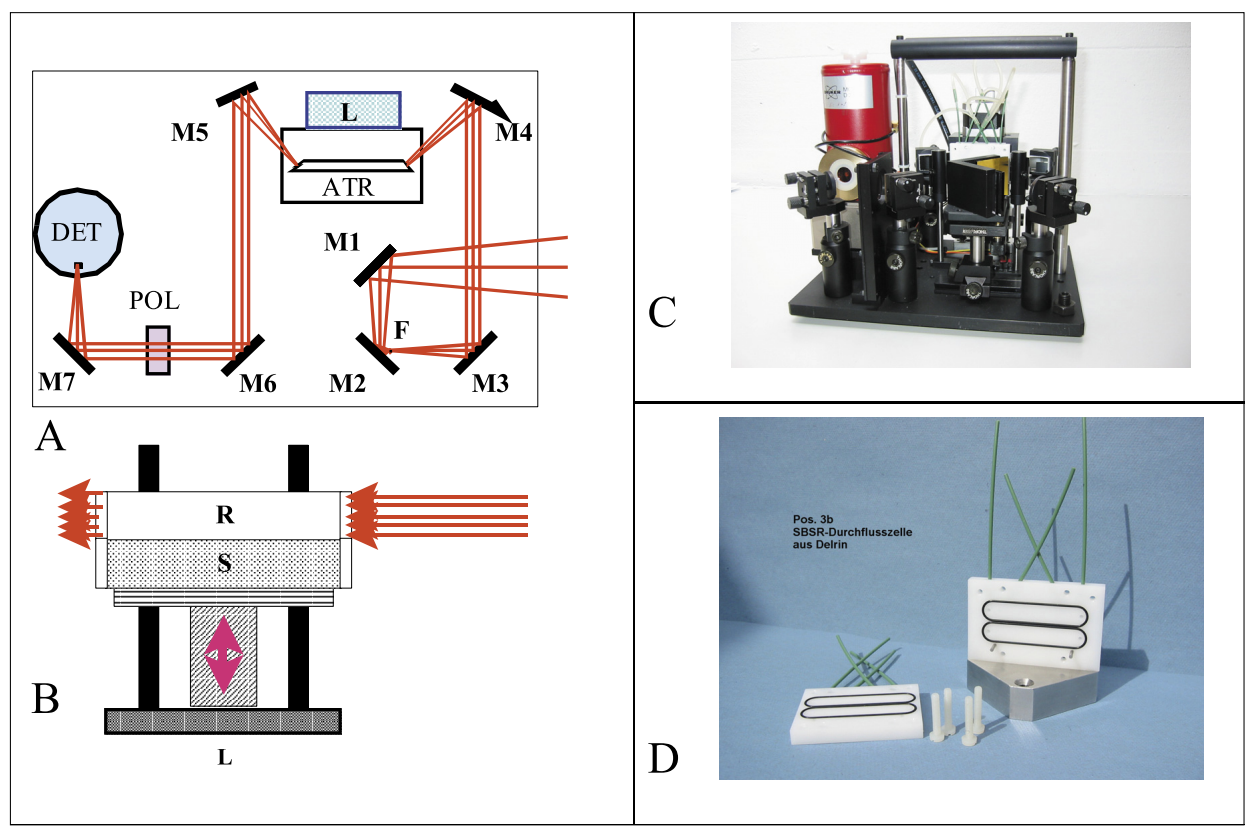

Fig. 1. Single-beam Sample Reference (SBSR) ATR attachment. (A) The focus in the sample compartment is displaced to the position $\mathrm{F}$ by the planar mirrors $\mathrm{M} 1$ and $\mathrm{M} 2$. The off-axis parabolic mirror M3 produces a parallel beam with a diameter of about one centimeter, i.e. half of the height of the multiple internal refection element (MIRE). The cylindrical mirror M4 focuses the light to the entrance face of the MIRE (ATR) which is mounted to the computer controlled lift L. M5 which has the same shape as M4 reconverts to parallel light passing via the planar mirror M6 through the polarizer POL and being focused to the detector DET by the off-axis parabolic mirror M7. (B) Alternative change from sample $S$ to reference $R$ and vice versa is performed by computer controlled lifting and lowering $L$ of the ATR cell body. (C) Photograph of a SBSR ATR attachment Mod. 3B [2] and (D) view into the interior of a SBSR ATR flow-through cell [2].

beam instrument. The principle features of this attachment are depicted in Fig. 1.

As usual, a convergent IR beam enters the sample compartment. Since the focal length of the focusing mirror, which is part of the FTIR instrument, is about four times longer than that of $\mathrm{M} 3$, this mirror will produce again a parallel beam, however, with a four times reduced diameter. By means of this attachment the focal point is displaced by the planar mirrors M1 and M2 to the position F. $\mathrm{F}$ is the focal point of the off-axis parabolic mirror M3, which performs a conversion of the divergent beam into a parallel beam with fourfold reduced cross-section. This beam is focused to the entrance face of a trapezoidal multiple internal reflection element (MIRE) by a cylindrical mirror M4. Therefore, the ray propagation in the MIRE is still parallel to the direction of light propagation (x-axis), enabling subdivision of the large MIRE surfaces (x, y-plane) in perpendicular direction (y-axis) to the light propagation. One half of the MIRE is then used for the sample (S) and the other one for the reference (R). Both, S and $\mathrm{R}$, were encapsulated by flow-through compartments, independently accessible by liquid or gaseous flow-through. This principle is referred to as the Single-beam Sample Reference (SBSR) technique [1][3].

The cell platform is moved cyclically up and down under computer control, thus aligning the sample and reference compartment alternatively with the IR beam. Therefore, SBSR absorbance spectra are calculated from sample and reference single channel spectra which have been measured with very short mutual time delay. Fig. 2 and Fig. 3 demonstrate the enhanced baseline stability of SBSR compared to the conventional SB technique, especially for long-term experiments. In order to enable an unambiguous comparison of the two techniques a clean germanium (Ge) MIRE was mounted on the SBSR attachment. Data acquisition was started a short time after closing the sample compartment, i.e. at a moment where purging with dry air was still not completed. The result is shown in Fig. 2.

Spectra $\mathrm{a}$ and $\mathrm{b}$ are conventional SB absorbance spectra. The corresponding reference spectra through the $\mathrm{R}$ and $\mathrm{S}$ channels (see Fig. 1B) were measured at the beginning of the experiment, whereas the sample spectra for each channel were measured after $1 \mathrm{~h}$. In this time interval purging progressed as recognized by atmospheric absorptions by water vapor and carbon dioxide. Spectrum Fig. 2c is the corresponding corrected SBSR spectrum. In practice SBSR spectra are recorded by successive movement of the MIRE up and down in a parallel IR beam. Fig. 2d, and 2e show the 


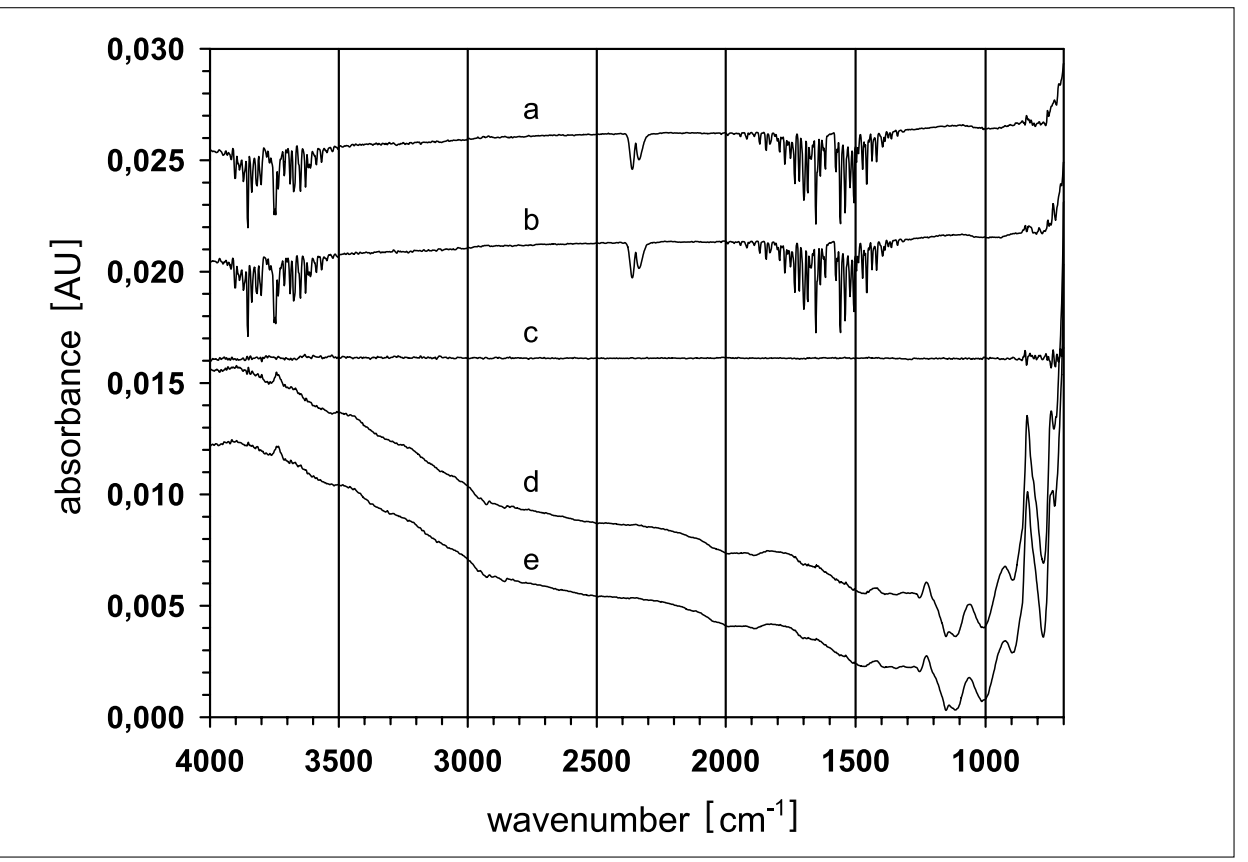

Fig. 2. FTIR ATR absorbance spectra of a clean Ge MIRE in dry air environment. (a) and (b) show the spectral changes resulting from water vapor and $\mathrm{CO}_{2}$ when conventional SC spectra are measured 60 min apart of each other in both channels, R and S, respectively. (c) Corrected SBSR spectrum. Al water vapor and $\mathrm{CO}_{2}$ absorption bands as well as inhomogeneities resulting from optical components are compensated because of the quasi-simultaneous data acquisition in both channels $(20(R, S)$ cycles with 50 scans per cycle) resulting in two averaged $R$ and $S$ single channel (SC) intensity spectra with 1000 scans each. (d) and (e) are the SBSR spectra (calculated by $A=-\lg (S / R))$ at the beginning of the experiment and after $60 \mathrm{~min}$, respectively. Both exhibit uneven baselines mainly as a consequence of chemical and geometrical inhomogeneities of the Ge MIRE. The corrected SBSR spectrum (c) is obtained as difference of spectra (e) and (d) which corresponds also to the difference of the single-beam SB absorbance spectra (b) and (a). However, one should be aware that the conventional SB mode does not offer the measurement of spectra in the $\mathrm{R}$ and $\mathrm{S}$ channel quas simultaneously, as depicted by Fig. 1. Experimental conditions: Ge MIRE; parallel polarized light; T $30{ }^{\circ} \mathrm{C}$; angle of incidence $\Theta=45^{\circ} ; \mathrm{N}=25$ internal reflections.

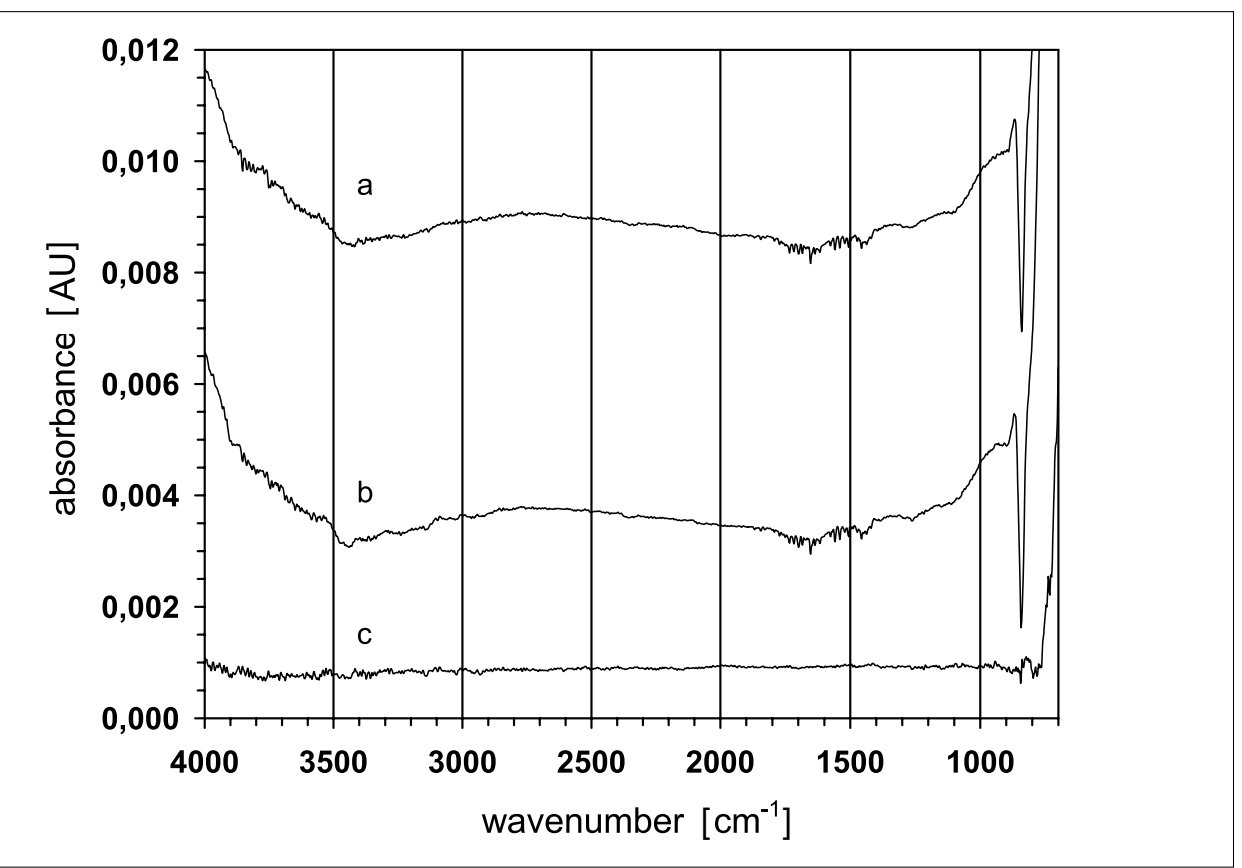

Fig. 3. FTIR ATR absorbance spectra of a clean Ge MIRE in dry air environment. (a) and (b) show the spectral changes by instrumental instabilities (e.g. re-warming of the MCT detector, water vapor incompensation) after 200 min measured in the $\mathrm{R}$ and in the S channel of the SBSR setup, respectively (Fig. 1). (c) SBSR absorbance spectrum. All atmospheric and instrumental instabilities are significantly better compensated because of the quasi-simultaneous data acquisition in both measuring channels (20 (R,S)-cycles with 50 scans per cycle) resulting in two average R and S intensity spectra with 1000 scans each. Experimental conditions: Ge MIRE; parallel polarized light; $\mathrm{T} \sim 30^{\circ} \mathrm{C}$; angle of incidence $\Theta=45^{\circ}, \mathrm{N}=25$ internal reflections. resulting absorbance spectra as obtained at the beginning of purging and after $1 \mathrm{~h}$. Some further relevant remarks should be made: (i) Since the Ge MIRE was clean in both cases, the spectra Fig. 2d and 2e should result in a flat baseline over the whole range of $\mathrm{Ge}$ transmittance. The experiment, however, shows that this is not the case. Inhomogeneity of the Ge crystal is one reason for these uneven baselines, and slight imprecision of the crystal shape the other. The latter may result in a slight displacement of the focus at the detector surface which affects also the baseline unless the detector exhibits a quite homogeneous sensitivity over the whole surface. Since this imperfection of optical components is constant for a given setup, it is generally recommended to measure a SBSR reference spectrum at the beginning of each experimental series. This spectrum is very stable over a long time period and can be used for the compensation of inhomogeneities introduced by optical components in all subsequent SBSR spectra of the series. As a consequence the difference between the SBSR spectra shown in Fig. 2d and 2e result in the corrected SBSR spectrum with a flat baseline. (ii) It should be noted that atmospheric disturbances by water vapor and carbon dioxide are automatically compensated by the SBSR principle as demonstrated by Fig. $2 d$ and 2e. (iii) Since the SB spectra shown in Fig. $2 \mathrm{a}$ and $2 \mathrm{~b}$ are a natural part of SBSR data acquisition, the spectrum of Fig. 2c is the difference between spectra Fig. $2 b$ and Fig. $2 a$.

In case of measurements with longer duration, instabilities of the FTIR spectrometer may also play a relevant role, especially when very low signals are to be detected. Fig. 3 shows the comparison of baseline stability with SB and SBSR technique when the measurements to be compared are made $200 \mathrm{~min}$ apart of each other. The experimental setup was the same as for data shown in Fig. 2. Fig. 3a and 3b are again the SB absorbance spectra, while Fig. $3 \mathrm{c}$ reflects the corrected SBSR absorbance spectrum, as explained earlier. The enhanced baseline stability is clearly demonstrated since all data are collected in the course of the same experiment, i.e. under absolutely the same conditions. All SBSR ATR equipment was obtained from OPTISPEC, Neerach (Switzerland) [2].

\subsection{Modulated Excitation Spectroscopy}

Time-resolved spectroscopy (TRS) can be used for kinetic studies of chemical reactions and can provide a new quality of information compared to experiments performed in the equilibrium state of a system. The possibility to achieve increased information about a system is unfortunately always paralleled by an increased experimental complexity. While slow reactions 
(in the second-range or slower) can be detected with almost every modern FTIRspectrometer by simply collecting spectra during the progression of the chemical reaction, more complex techniques like stepscan spectroscopy have to be used for the investigation of faster processes. Reversible processes or processes which can be repeated multiple times are a prerequisite for all time-resolved measurement techniques with a time resolution smaller than about 10-100ms (mainly dependent on the desired spectral resolution).

The absorbance changes of functional groups involved in the observed chemical reaction are in general much smaller than the 'stationary' absorbance of the group and therefore much harder to detect.

Modulated excitation (ME) combined with adequate data processing can help to detect these small signal changes within a noisy or a large background. Moreover, kinetic parameters of stimulated reactions can be determined analogously as with relaxation experiments using a jump excitation via an external parameter [4]. The advantages of ME compared to excitation using a fast transfer of an external parameter are: (i) the possibility to separate different kinetic time constants by the choice of the frequency of the ME, (ii) the possible spectroscopic separation of components that are responding with different time constants and therefore at different phases to the ME by the use of phase sensitive detection (PSD), and (iii) the enhanced signal-to-noise ratio achieved by the PSD due to the elimination of all signal components which do not have the same frequency as the excitation itself.

Modulation techniques always need a ME of a sample followed by a PSD of the measured quantity [5]. It can only be applied to systems that show a (pseudo-) reversible response to a periodic excitation. The whole range of physical-chemical parameters can be used as excitation parameters, such as temperature [6][7], electric field [4][8][9], reactant concentration [10-15], $\mathrm{pH}$ value [4], mechanical force [16], pressure [17], or radiant power [18].

Every reversible reaction responds to a periodic stimulation with periodic variations of all influenced molecular parts. This periodic response shows in general predominately the same frequency as the excitation (Fig. 4). However, depending on the shape of the excitation function and on the type of chemical reaction, the response can also include multiples of the stimulation frequency. As an example, a reaction scheme consisting only of first order elementary steps acts as a linear system and reproduces the frequency spectrum of the excitation function. However, if the chemical reaction scheme contains non-linear steps, in the simplest case a second order reaction, its response to a harmonic exci-

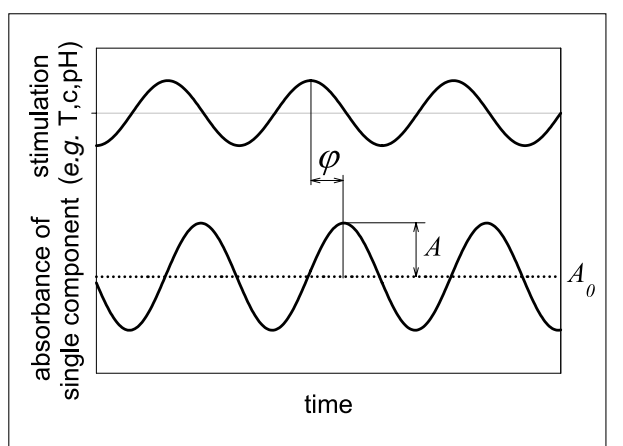

Fig. 4 Modulated excitation (ME) and modulated response. If a reversible system is excited by a periodic stimulation (e.g. temperature, particle concentration, $\mathrm{pH}$-value), all effected components will respond with absorbance changes showing the same frequency and/or higher harmonics. The variation of the absorbance is always located around a mean absorbance $A_{0}$ which may be regarded as background that is often much larger than the modulated absorbance amplitude $\mathrm{A}$. The phase lag $\varphi$ can be considered as the time difference between maxima (or minima) of the stimulation and of the response function expressed in radians or degree. Both, the modulated absorption amplitude $A$ and the phase lag $\varphi$ of the absorption at the used stimulation frequency give information on kinetic properties of the system. Experimentally they are obtained by phase sensitive detection (PSD) of the spectroscopic data.

tation will contain also higher harmonics which are characteristic of the whole reaction scheme.

In FTIR ME spectroscopy, PSD of the measured IR intensity provides the changes of the absorbance in the measured spectral region in the form of phase-resolved spectra. From those phase-resolved spectra we can evaluate the parameters of the chemical reaction, i.e. the amplitudes and phase lags of all absorption bands corresponding to functional groups involved in the chemical reaction. For the study of kinetic properties, this can be done for the fundamental excitation frequency as well as for higher harmonics. PSD of complex sample response thus enables selective detection of only those signals exhibiting the frequency of excitation and multiples of it. Each of them is characterized by amplitude and phase lag with respect to stimulation. In the simplest case of modulation spectroscopy, the frequency of the excitation function is slow compared to the time constants of the observed process. In such experiments the only advantage of ME is the enhanced signal-to-noise ratio achieved by the PSD. The resulting phase-resolved spectra can then be read like normal difference spectra. Although the evaluation of kinetic constants is not possible in such experiments, they can be used for the detection of very small signal changes that could not be determined by normal difference spectroscopy due to a large background or a noisy environment. In section 3.2.1. we will show such an experiment using ME to detect and quantify a $250 \mu \mathrm{AU}$ signal change within a $25 \mathrm{mAU}$ background.

So far, ME spectroscopy appears as a special type of difference spectroscopy. The real potency of ME spectroscopy is revealed however when the period of the stimulation frequency is in the order of magnitude of the kinetics of the stimulated system, i.e. if one or more relaxation times of the externally excited reaction fulfill the condition $0.1<\omega \tau_{i}<10$, where $\omega$ denotes the angular frequency of the stimulation and $\tau_{i}$ is the relaxation time of the $\mathrm{i}$-th component in the system. In this case significant phase lags $\varphi_{i}$ between stimulation and sample responses will occur. For a simple first order kinetic, phase lag, relaxation time, and stimulation frequency are related as shown in Eqn. (1) [4].

$$
\varphi_{i}=\operatorname{atan}\left(-\omega \cdot \tau_{\mathrm{i}}\right)
$$

An increasing phase shift with increasing modulation frequency is always paralleled by a decrease of the modulated absorption amplitude of the component $\mathrm{A}_{\mathrm{i}}$. Both are significant of the underlying reaction scheme and the associated rate constants of the stimulated process [19].

The PSD is the tool that enables us to evaluate the absorption amplitude $A_{i}$ and the phase lag $\varphi_{i}$ of different components $i$ from our spectroscopic data. The mathematical fundament for PSD in spectroscopy can be found in [4] and [5]. It should be noted that the output of the PSD - the socalled phase-resolved spectrum - depends on the user-selectable phase $\Phi^{P S D}$ of the PSD. The phase-resolved absorbance $\mathrm{A}_{i}^{\Phi^{P S D}}$ for a single component i (vibrational mode) is given by Eqn. (2).

$$
A_{i}^{\phi^{P S D}}=A_{i} \cos \left(\varphi_{i}-\phi^{P S D}\right)
$$

Regarding the maximum and the minimum of the cosine function in Eqn. (2), we get the maximum and the minimum output of the PSD at $\phi^{P S D}=\varphi_{i}$ and $\phi^{P S D}=\varphi_{i} \pm 90^{\circ}$ respectively (Eqn. (3)).

$$
\begin{aligned}
& A_{i, \text { max }}^{\phi^{P S D}}=A_{i} \text { for } \phi^{P S D}=\varphi_{i} \\
& A_{i, \text { min }}^{\phi^{P S D}}=0 \quad \text { for } \quad \phi^{P S D}=\varphi_{i} \pm 90^{\circ}
\end{aligned}
$$


If the absorption band of the component $i$ does not overlap with other absorption bands, the modulated absorption amplitude and the phase lag of this species can therefore immediately be found by maximizing or minimizing the output of the PSD in given a wavenumber range by the variation of $\phi^{P S D}$.

Moreover, in case of overlapping absorption bands, the selectivity of the PSD enables us to separate components with different kinetic properties. Let us assume two different absorbing components $\mathrm{A}_{1}$ and $\mathrm{A}_{2}$ excited by an appropriate stimulation frequency and therefore showing different phase lags $\varphi_{1}$ and $\varphi_{2}$, respectively (Eqn.(1)). The phase-resolved absorption $\mathrm{A}_{i}^{\Phi^{P S D}}$ of component 1 vanishes in the phase-resolved spectrum when we select $\Phi_{1}^{P S D}=\varphi_{1} \pm 90^{\circ}$ (Eqn.(3)). Now, this phase-resolved spectrum only contains the absorption of component 2, which is not zero because of $\varphi_{1}$ $\neq \varphi_{2}$, as assumed. The optical properties (maximum of peak-position and full width at half maximum) of component 2 can now be determined without any further assumptions. In the same way the physical properties of component 1 can be determined by selecting $\Phi_{2}{ }^{P S D}=\varphi_{2} \pm 90^{\circ}$, which eliminates the absorbing parts of component 2. Additionally to the band separation we naturally get the absolute phase lags and the modulation amplitudes of the components at the used stimulation frequencies. The dependence of the phase lag and the amplitude on $\omega$ may be calculated based on a given reaction scheme. Analytical expressions for a simple reversible reaction $A 1<=>A 2$ are given in [4].

If a set of absorption bands exhibits the same phase lag $\varphi_{i}$ it is considered as a correlated population. Such a population consists, e.g. of molecules or parts of them that are involved in the same reaction step. The assignment of a group of absorption bands in a modulation spectrum to a population is considered to be validated if upon changing the stimulation frequency $\omega$ all of these bands exhibit further on the same dependence with respect to phase lag $\varphi_{i}$ and amplitude $A_{i}$. Obviously, ME spectroscopy enables a very rigorous test of the significance of a reaction scheme since consistence of experimental data with theory derived from a given reaction scheme must hold on over the whole frequency range of stimulation.

As an example for the capability of MEspectroscopy we present the band separation of the strongly overlapping absorption of a polypeptide (PLL) in the amide I' region.

\section{Applications}

\subsection{SBSR Spectroscopy}

3.1.1. Interaction of LPS with a DPPA/ (POPC:HDPyr) Bilayer

In [20], the interaction of negatively charged lipopolysaccharide (LPS) from
Pseudomonas aeruginosa (serotype 10) with different kinds of surfaces was investigated by FTIR ATR spectroscopy. Here, we will present the interaction of LPS with a positively charged lipid bilayer consisting of a dipalmitoyl phosphatidic acid (DPPA) inner monolayer attached to the Ge MIRE and an outer monolayer consisting of equal molar amounts of palmitoyl oleoyl phosphatidyl choline (POPC) and hexadecyl pyridinium (HDPyr) as positively charged component.

Fig. 5 shows the influence of three different LPS concentrations on the bilayer, which was attached to a Ge MIRE. Three absorbance spectra are shown for each concentration. The lowest spectrum of each group reflects the absorbance of the bilayer in contact with LPS, and is referred to as sample $\mathrm{S}$. The middle spectrum shows the absorbance from the bilayer in contact with pure buffer, and is therefore referred to as reference R. Finally, the upper trace of each group denotes the actual difference spectrum $\mathrm{S}-\mathrm{R}$, which is referred to as SBSR. The reference spectra used to calculate the $\mathrm{S}$ and $\mathrm{R}$ absorbance spectra were recorded before the experiment with the lowest LPS concentration. S and R compartments were separately accessible via tubes, enabling the preparation of the DPPA/(POPC:HDPyr) bilayer at the same time in the $\mathrm{R}$ and $\mathrm{S}$ compartment, however, exposing only the sample $\mathrm{S}$ to LPS while $\mathrm{R}$ remains in contact with buffer. Thus SBSR spectra reflect the instantaneous difference between (POPC:HDPyr)/LPS and (POPC: HDPyr)/buffer, while $\mathrm{S}$ and $\mathrm{R}$ absorbance spectra report on the whole history of LPS and buffer interaction, respectively, since the measurement of the corresponding reference spectra was performed at the beginning of the experimental series.

The negative $v(\mathrm{CH})$ bands of the R spectra in Fig. 5 show unambiguously that also the DPPA/(POPC:HDPyr) bilayer in contact with pure buffer was not absolutely stable during the time course of the experiment. However, at all LPS concentrations, the S spectra reflected a significantly higher lipid loss than the R spectra, which is obviously a consequence of LPS interaction with the positively charged bilayer. Assuming approximately equal spontaneous lipid loss in $\mathrm{R}$ and $\mathrm{S}$, the SBSR spectra reflect the lipid loss resulting predominantly from LPS interaction. As further qualitative and quantitative studies showed, LPS has extracted positively charged HDPyr lipids from the outer monolayer of the membrane [20].

This example illustrates the necessity of recording a quasi-simultaneous reference to quantify the effect of a specific interaction, if the sample is not absolutely stable over a longer period of investigation. In a conventional single-beam (SB) experiment only the total loss of lipid during the experiment is measured. Therefore, it is impossible to quantify within a single experiment which

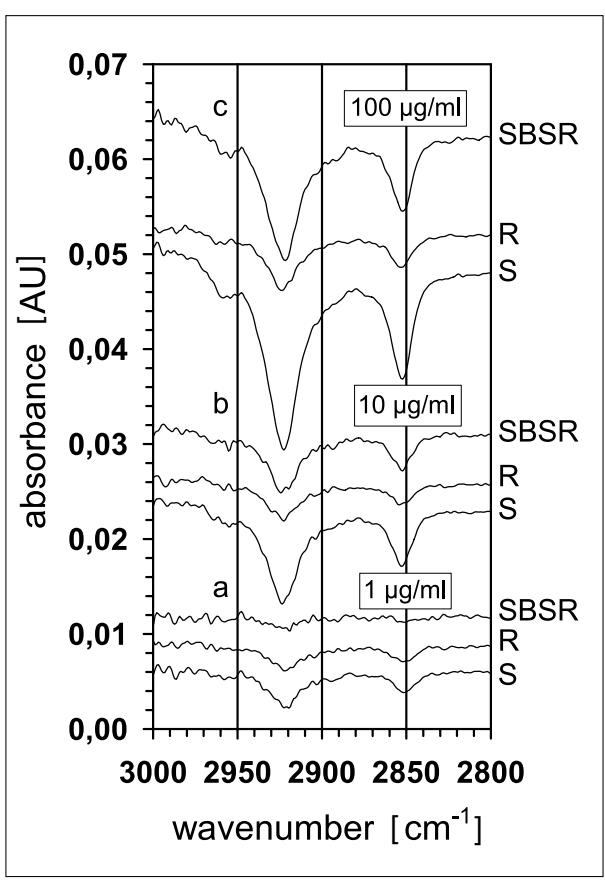

Fig. 5. Interaction of a lipopolysaccharide (LPS) with a bilayer membrane consisting of dipalmitoyl phosphatidic acid (DPPA), palmitoyl oleoyl phosphatidyl choline (POPC), and the positively charged hexadecyl pyridinium (HDPyr). FTIR ATR absorbance spectra of a DPPA/(POPC: HDPyr) bilayer interacting with (a) $1 \mu \mathrm{g} / \mathrm{ml}$, (b) 10 $\mu \mathrm{g} / \mathrm{ml}$, (c) $100 \mu \mathrm{g} / \mathrm{ml}$ LPS measured after $30 \mathrm{~min}$ of interaction with LPS in each case. Reference: DPPA/(POPC:HDPyr) bilayer in contact with pure buffer. S, R: Absorbance spectra measured in the SB mode in the sample and reference channel of the SBSR ATR flow-through cell, respectively. Single channel reference spectra were stored before beginning of LPS application in the S-channel. SBSR: Difference absorbance spectra $S-R$ accumulated in the SBSR mode. SBSR spectra reflect the actual difference between a sample and a reference of quasi the same age, since the DPPA/(POPC:HDPyr) membranes have been prepared at the same time and by the same procedures in the S- and R-compartment. The negative $\mathrm{CH}_{2}$ stretching bands at $\sim 2920 \mathrm{~cm}^{-1}$ and $\sim 2850 \mathrm{~cm}^{-1}$ in the Rchannel reveal a continuous loss of lipids during the time course of the experiment, probably due to electrostatic repulsion between positively charged HDPyr molecules. A significantly higher loss was observed, however, in presence of LPS as revealed by S-channel SB and SBSR spectra. Experimental conditions: Ge MIRE; parallel polarized light; $\mathrm{T}=25^{\circ} \mathrm{C}$; angle of incidence $\Theta$ $=45^{\circ}$; number of active internal reflections $\mathrm{N}=$ $36.6 \pm 1$. Buffer: $20 \mathrm{mM}$ phosphate $\mathrm{pH} 7.0,100$ $\mathrm{mM} \mathrm{NaCl}$.

portion of this loss is caused by the presence of LPS and which one by spontaneous desorption.

\subsubsection{Interaction of TNF $\alpha$ with an Anti- body}

The interaction of TNF $\alpha$ with an antibody was investigated in [21]. The main 
a sample compartment

b reference compartment

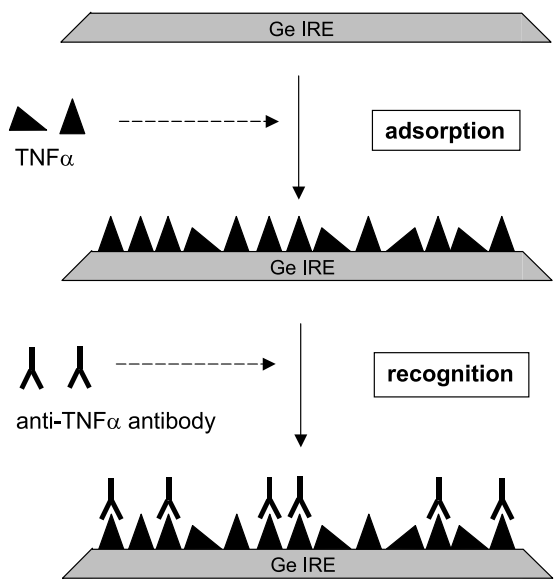

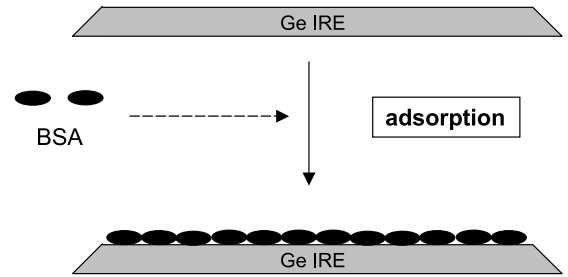

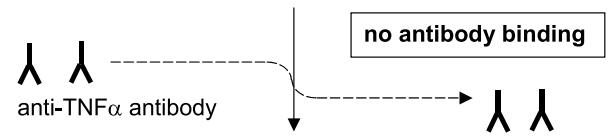

Ge IRE

Fig. 6. Interaction of anti-TNF $\alpha$ antibody with an immobilized monolayer of TNF $\alpha$. Schematic description of the pathways of the experiments performed in the sample and reference channels of the SBSR flow-through cell (Fig. 1d). Each step was monitored by FTIR ATR spectroscopy. The corresponding absorbance spectra are shown in Fig. 7. Steps of buffer rinsing after protein adsorption and antibody binding are not shown. (a) Pathway in the sample (S) channel: After adsorption of TNF $\alpha$ to the Ge MIRE (Fig. 7a), antibody for recognition of TNF $\alpha$ was added (Fig. 7b). (b) Pathway in the reference (R) channel: After adsorption of BSA to the Ge MIRE (Fig. 7c), antibody was added to ensure that no recognition of BSA and no unspecific binding to the Ge surface took place (Fig. $7 \mathrm{~d}$ ).

7. The S compartment spectra (Fig. 7a) and (Fig. 7b) show the absorbances of immobilized TNF $\alpha$ and of the AB bound to it, respectively. The former acted as a reference for the latter. The $\mathrm{R}$ compartment spectrum (Fig. 7c) shows the signal of the immobilized BSA, and from Fig. 7d can be concluded that no unspecific binding of $\mathrm{AB}$ took place. Besides qualitative studies, the surface concentrations $\Gamma$ of all used proteins were determined and indicated in the caption of Fig. 7.

The advantage of the SBSR setup in this experiment is the possibility to offer the $A B$ simultaneously to two different surfaces which enables studies of specific interactions under very same conditions of external parameters and reactant age.

\subsubsection{Formation of an Oriented Mono-} layer of Omp32 by in situ Dialysis [22]

The development of a reproducible technique for the preparation of oriented porin Omp32 monolayers on an ATR multiple internal reflection element (MIRE) was the principal aim of this work. A procedure earlier applied for 2-dimensional crystallization of membrane proteins could targets of these experiments were to find a method to produce a stable and functional immobilized human tumor necrosis factor$\alpha(\mathrm{TNF} \alpha)$ layer on a Ge MIRE and to unambiguously verify the interaction of this immobilized layer with an antibody (AB) by in situ FTIR ATR spectroscopy. A schematic representation of the sequence of experimental steps in the $\mathrm{R}$ and $\mathrm{S}$ compartments is given in Fig. 6. At first, both the $\mathrm{R}$ and $\mathrm{S}$ compartments were filled and rinsed with phosphate buffer saline (PBS) and reference spectra were recorded. Afterwards, $\mathrm{TNF} \alpha$ solution was pumped into the $\mathrm{S}$ compartment only, the R compartment was treated in the same way with PBS. After the TNF $\alpha$ adsorption to the Ge MIRE had taken place, $\mathrm{R}$ and $\mathrm{S}$ compartments were rinsed with PBS. After acquiring spectra of immobilized TNF $\alpha$, only the R compartment was saturated by a concentrated solution of bovine serum albumin (BSA) $(25 \mathrm{mg} /$ $\mathrm{ml}$ PBS). Then, in the same way, all compartments were rinsed, and after recording spectra of the $\mathrm{S}$ side ( $\mathrm{S}$ compartment with immobilized $\mathrm{TNF} \alpha$ ) and of the R side ( $\mathrm{R}$ compartment with immobilized BSA), a solution of anti-TNF $\alpha$ antibody (AB) (100 $\mu \mathrm{g} / \mathrm{ml}$ in PBS) was pumped into both $\mathrm{R}$ and $\mathrm{S}$ compartments. After a last washing step, spectra of $\mathrm{AB}$ bound to immobilized TNFa in the S compartment and of immobilized BSA in the R compartment, where no bound $\mathrm{AB}$ was detected, were acquired.

Each step in Fig. 6 is documented by corresponding absorbance spectra in Fig.

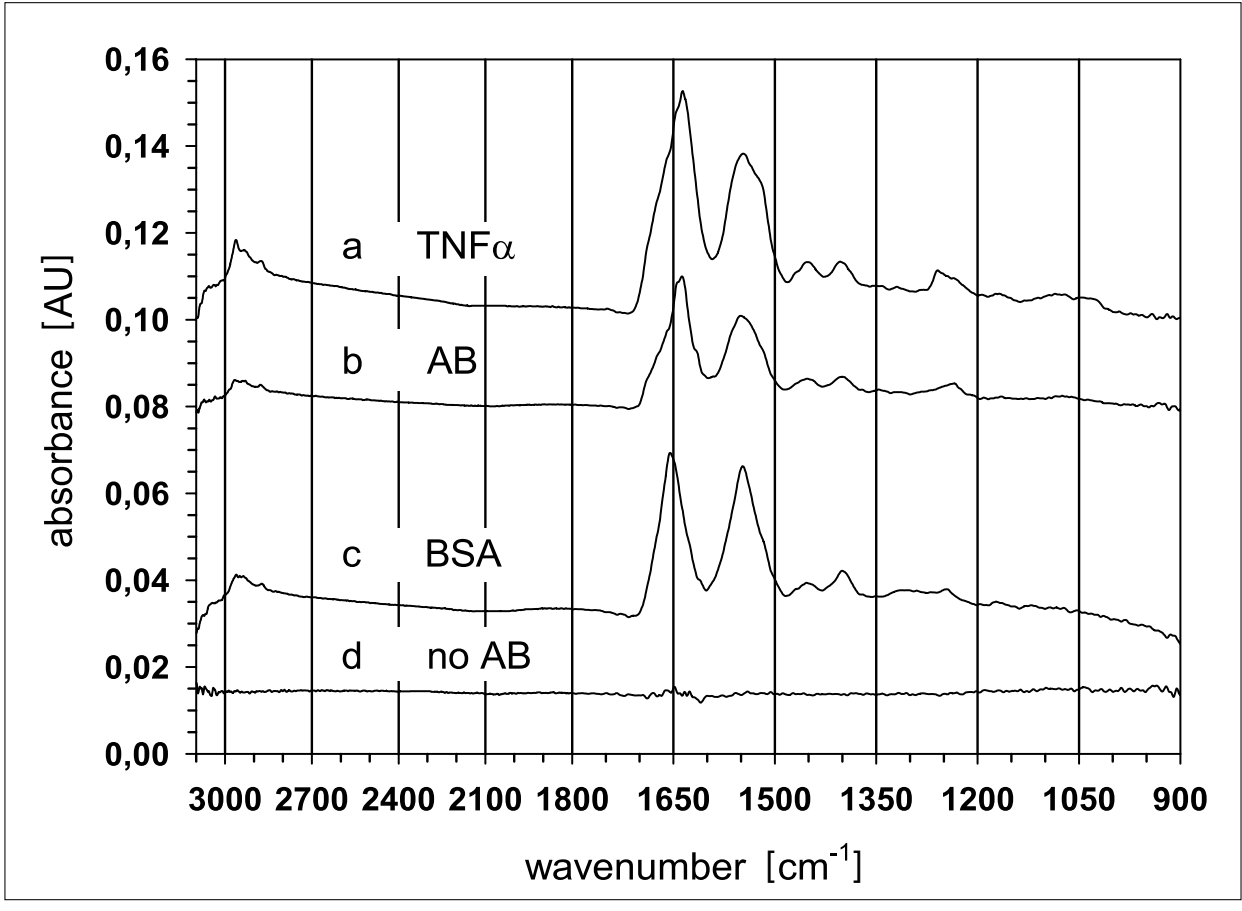

Fig. 7. IR ATR absorbance spectra of immobilized TNF $\alpha$, immobilized BSA and anti-TNF $\alpha$ antibody (AB) bound to TNF $\alpha$. (a) TNF $\alpha$ adsorbed to the Ge MIRE in the S channel of the flow-through cell, with the Ge MIRE in contact with PBS as reference; surface concentration $\Gamma_{\mathrm{TNF} \alpha}=(7.51 \pm 0.66) \cdot 10^{-12}$ $\mathrm{mol} \mathrm{cm}{ }^{-2}$. (b) Anti-TNF $\alpha$ AB bound to an adsorbed TNF $\alpha$ monolayer on the Ge MIRE in the S channel, with TNF $\alpha$ adsorbed to the Ge MIRE as reference; surface concentration $\Gamma_{A B}=(1.41 \pm 0.12) \cdot 10^{-12}$ $\mathrm{mol} \mathrm{cm}^{-2}$. (c) BSA adsorbed to the Ge MIRE in the R channel of the flow-through cell, with the $\mathrm{Ge}$ MIRE in contact with PBS as reference; surface concentration $\Gamma_{\mathrm{BSA}}=(5.70 \pm 0.49) \cdot 10^{-12} \mathrm{~mol} \mathrm{~cm}^{-2}$. (d) Difference spectrum between immobilized BSA in the $R$ channel after and before rinsing with $A B$ solution. In contrast to (b), no detectable binding of the anti-TNF $\alpha$ AB to BSA was found. Surface concentrations $\Gamma$ were calculated from the integrated intensities of the amide II bands at $\sim 1550 \mathrm{~cm}^{-1}$. Experimental conditions: Ge MIRE; parallel polarized light; $\mathrm{T}=25^{\circ} \mathrm{C}$; angle of incidence $\Theta=45^{\circ}$; number of active internal reflections $\mathrm{N}=26.8 \pm 1$. Buffer: $10 \mathrm{mM}$ phosphate $\mathrm{pH} 7.6,100 \mathrm{mM} \mathrm{NaCl}$, $0.02 \%(\mathrm{w} / \mathrm{v})$ sodium azide. 
be successfully adapted [23]. Layer formation was performed in a spectroscopic dialysis cell [2] in presence of 1,2-dimyristoyl-sn-glycero-3-phosphocholine (DMPC) micelles by the removal of the detergent (OPOE). This setup enabled in situ monitoring of the monolayer formation by means of FTIR single-beam sample reference (SBSR) attenuated total reflection (ATR) technique. The time course of OPOE and sodium azide extraction by dialysis as well as the formation of the protein/lipid layer on top of the IRE was evaluated from corresponding time-dependent absorbance changes in the IR spectra. Both OPOE and azide featured first order kinetics, whereas the synchronous adsorption of porin to the Ge MIRE resulted in a sigmoidal time behavior. At the beginning of the dialysis, the concentration of OPOE was just above the critical micellar concentration $(\mathrm{cmc})$ which is about $0.25 \%$. As the $\mathrm{cmc}$ was reached by dialysis, Omp32 adsorption was accelerated, reaching saturation after about $5 \mathrm{~h}$. Quantitative analysis of the Omp32/lipid layer gave strong evidence for a well ordered monolayer with the barrel axis of the Omp32 trimer being approximately oriented perpendicular to the supporting MIRE. Two to six DMPC molecules were detected per Omp32 monomer.

\subsection{Modulation Spectroscopy}

3.2.1. Binding of Sodium Cholate to an Adsorbed Layer of Human Serum Albumin (HSA)

HSA is the most abundant protein in blood with a concentration of $5 \mathrm{~g} / 100 \mathrm{ml}$. The main structural element of HSA is the $\alpha$-helix (67\%), the amount of $\beta$-forms is $10 \%$ [24]. Cholate is a ligand of HSA and belongs to the group of bile acids. It was reported earlier by equilibrium dialysis that HSA in solution has 2.8 binding sites to cholate with a binding constant of 3300 and 12.0 binding sites with a binding constant of 300 [25]. The aim of this study was to determine the number of cholate molecules that are able to bind to one adsorbed albumin molecule and to investigate how bound cholate molecules influence the secondary structure of the adsorbed albumin.

Since utmost sensitivity is required to reach this aim, concentration-modulated excitation (c-ME) was the experimental method of choice (Fig. 8). C-ME is performed by periodic alteration of the concentration of an effector molecule in the SBSR flowthrough cuvette (Fig. 1d). Experimentally this was performed by two computer-controlled pumps. During the first half-period a $10 \mathrm{mM}$ solution of sodium cholate in buffer was pumped through the cuvette, while during the second half-period the cuvette was flushed by pure buffer solution (10 $\mathrm{mM} \mathrm{Na} \mathrm{HPO}_{4} / \mathrm{NaH}_{2} \mathrm{PO}_{4} \mathrm{pH} 7.2,150 \mathrm{mM}$ $\mathrm{NaCl}$, and $0.02 \%$ (w/v) sodium azide), both

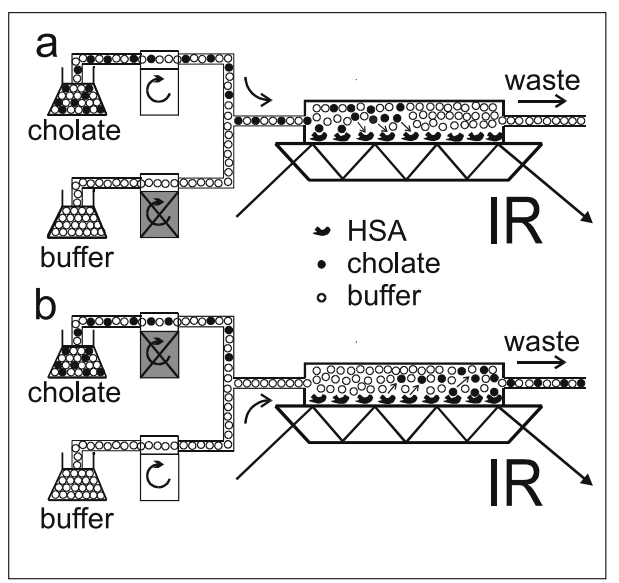

Fig. 8. Schematic setup for a concentrationmodulated excitation (c-ME) experiment [26] studying specific interaction between immobilized human serum albumin (HSA) and sodium cholate. (a) During the first half-period cholate is pumped through the ATR flow-through cell containing an adsorbed monolayer of HSA. (b) During the second half-period the cholate is washed out by pumping only buffer solution through the cell.

with the same flow rate of $1 \mathrm{ml} / \mathrm{min} .96$ successive periods of $9.6 \mathrm{~min}$ each were averaged to improve the signal-to-noise ratio. For details on data acquisition and manipulation the reader is referred to [5].

Fig. 9b represents the SBSR spectrum of a $10 \mathrm{mM}$ solution of sodium cholate in buffer. The spectrum is dominated by the asymmetric and symmetric stretching vibrations of the carboxylate group near 1550 and $1400 \mathrm{~cm}^{-1}$, respectively. The slightly negative $\mathrm{H}_{2} \mathrm{O}$ bending band at $1640 \mathrm{~cm}^{-1} \mathrm{re}-$ sults from water displacement by dissolved cholate, since pure buffer solution was in the $\mathrm{R}$ cuvette of the SBSR cell. The next step in this series was c-ME of sodium cholate in the $S$ cuvette of the cell, consisting of the uncoated Ge MIRE. The result is shown in Fig. 9c and d. These spectra are orthogonal, i.e. they are described by Eqn. (3). The fact that total signal canceling occurred in spectrum 9d indicates that no time-dependant processes occur with relaxation times in the 10 min range, as to be expected in this case. The spectra in Figs. 9c and 9d serve in the following as reference modulation spectra for c-ME of adsorbed HSA by cholate. In the next experimental step HSA was adsorbed to the clean Ge MIRE surface. This was performed by pumping a solution of albumin dissolved in buffer (conc: $20 \mathrm{mg} /$ $\mathrm{ml})$ through the SBSR sample (S) cuvette leading to spontaneous adsorption of the protein. After reaching equilibrium, the HSA solution was replaced by buffer. This procedure led to a slight HSA loss. Therefore rinsing with buffer was continued until the surface concentration of HSA remained constant. The corresponding SBSR ATR spectrum measured with parallel polarized incident light is shown in Fig. 9a. For the sake of comparison with the other spectra in this figure it was scaled down by a factor of 5. From the integrated absorbance of the amide II band near $1550 \mathrm{~cm}^{-1}$ a surface concentration of $(5.72 \pm 0.54) \cdot 10^{-12} \mathrm{~mol} / \mathrm{cm}^{2}$ was calculated in good agreement with the theoretical value of $(5.49 \pm 0.48) \cdot 10^{-12} \mathrm{~mol} /$ $\mathrm{cm}^{2}$ for a compact HSA monolayer. This value was calculated from crystallographic data [27] assuming a layer thickness of $29.5 \pm 2.5 \AA$. Now c-ME of this adsorbed albumin layer by cholate was performed in the same way as earlier with the clean Ge MIRE. The corresponding modulation spectrum measured with parallel polarized light and $\phi^{P S D}$ setting for maximum amplitude is shown in Fig. 10b. This spectrum contains the modulated responses of the HSA layer to the periodic interaction with cholate, and of the modulated concentration change of cholate in the bulk. However, the difference between spectra $10 \mathrm{~b}$ and $10 \mathrm{a}$ results in $10 \mathrm{c}$, i.e. the response of adsorbed HSA to cholate interaction. It should be noted that the absorbance of the bulk solution in contact with HSA is somewhat lowered compared to that in contact with the pure Ge-MIRE because of the displacement by the HSA layer. The effect is very small in the $1 \%$ region, but it was taken into account when the difference modulation spectrum $10 \mathrm{c}$ was calculated. Finally the expanded amide I and II region of the modulation spectra of HSA measured with polarized incident light are shown in Fig. 11. The two dominant positive bands at $1545 \mathrm{~cm}^{-1}$ and $1405 \mathrm{~cm}^{-1}$ reflect now $v_{\mathrm{as}}\left(\mathrm{COO}^{-}\right)$and $\mathrm{v}_{\mathrm{s}}\left(\mathrm{COO}^{-}\right)$of HSA bound cholate, respectively. Quantitative evaluation of the latter resulted in for the cholate surface concentration $(4.67 \pm 0.82) \cdot 10^{-11} \mathrm{~mol} /$ $\mathrm{cm}^{2}$, which corresponds to $8.4 \pm 0.6$ cholate molecules bound to one adsorbed HSA molecule at a cholate bulk concentration of $10 \mathrm{mM}$. Moreover there is a significant rather narrow band at $1656 \mathrm{~cm}^{-1}$. It appears in-phase with the two carboxyl stretching vibrations, thus indicating unambiguously that it is a direct consequence of cholate binding to HSA. A reasonable interpretation of this observation is the increase of $\alpha$-helical structures induced by cholate binding. More detailed information on this obvious secondary structural change will be given in forthcoming experiments, using $\mathrm{D}_{2} \mathrm{O}$ as a solvent. This will significantly simplify the situation in the important amide I region, because in case of $\mathrm{H}_{2} \mathrm{O}$ as solvent, it will never be possible to compensate its bending absorption band near $1640 \mathrm{~cm}^{-1}$ accurately due to molecule specific frequency shifts of bound water as well as due to water displacement by solutes in the bulk and by an adsorbed layer. Detailed results of this work will be published elsewhere (N. Hassler et al. 2005). 

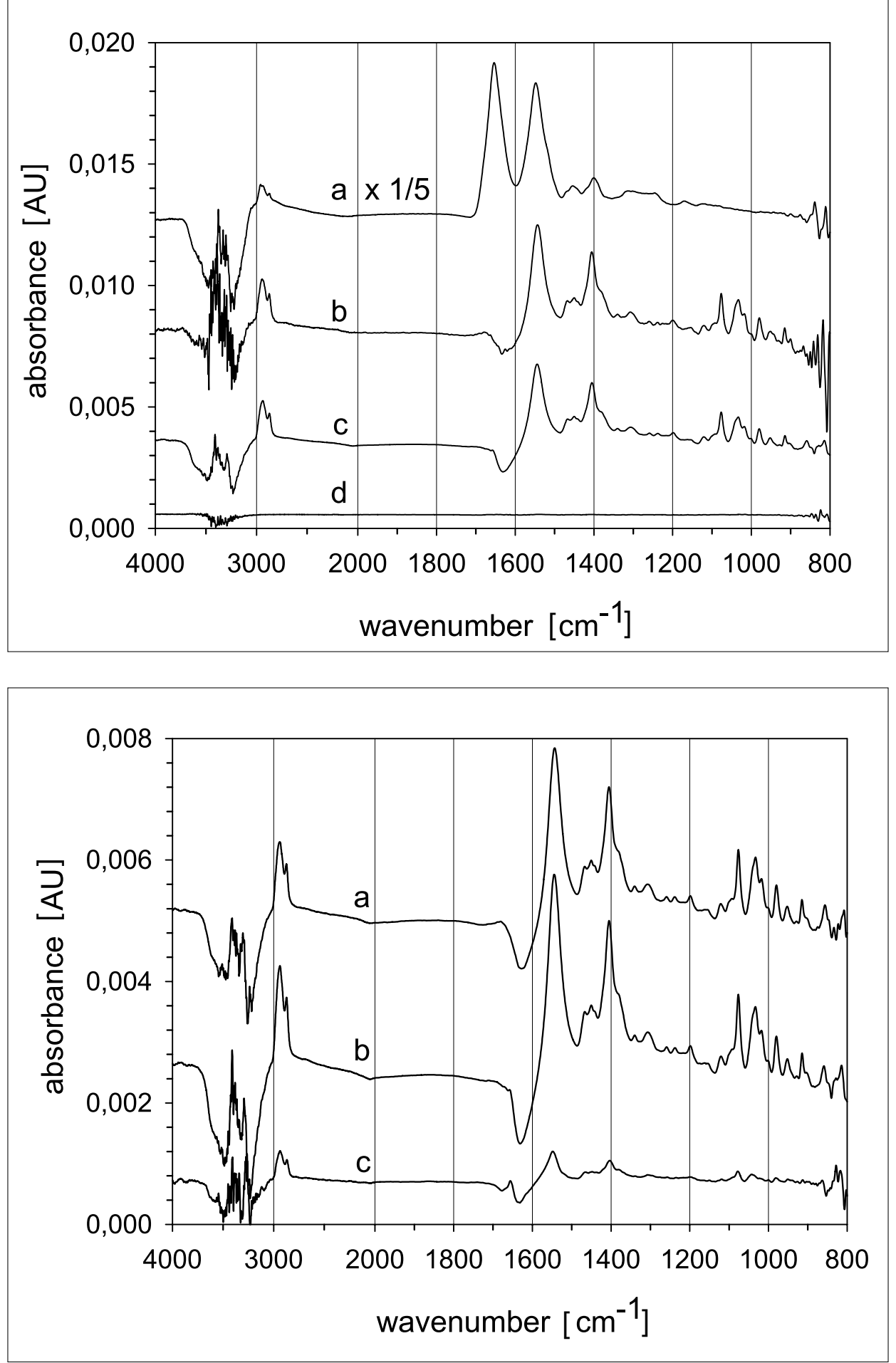

3.2.2. Structural Changes of Poly-LLysine (PLL) Induced by Temperature Modulated Excitation (T-ME)

The secondary structure of poly-L-lysine (PLL) can be reversibly converted from an $\alpha$-helix to an anti-parallel $\beta$ pleated sheet by T-ME [6]. In this T-ME experiment, a thin layer of PLL was exposed to an atmosphere of $80 \%$ relative humidity at a mean temperature of $26{ }^{\circ} \mathrm{C}$. A periodic variation of the temperature about this mean value by $\pm 2{ }^{\circ} \mathrm{C}$ with a period of $60 \mathrm{~s}$ was applied. Phase-sensitive detection with the fundamental and first harmonic frequency resulted in $0^{\circ}$ and $90^{\circ}$ phase-resolved FTIR modulation spectra. The amide I and II range is shown in Fig. 12. Both, the amide I and amide II vibrations are sensitive to protein and polypeptide secondary structures; however, since frequency shifts are generally small and a large quantity of amide groups are contributing, these bands are in most cases heavily overlapped. The reliable separation into components in the amide I and amide II region is a prerequisite for the analysis of a folding process in a protein or polypeptide.
Fig. 9. Cholate concentration-modulated excitation (c-ME). For the sake of comparison the ATR spectrum of a HSA monolayer measured with parallel polarized (pp) incident light was scaled down by a factor of 5 and shown in (a). The HSA surface concentration was calculated from the integrated absorbance of the amide II band to be $(5.72 \pm 0.54) \cdot 10^{-12} \mathrm{~mol} / \mathrm{cm}^{2}$, corresponding to a packed monolayer. (b) represents the SBSR ATR spectrum of a $10 \mathrm{mM}$ solution of sodium cholate also in pp polarization. (c) and (d) show c-ME spectra of a $10 \mathrm{mM}$ cholate solution as measured with the uncoated Ge MIRE. The phase setting of the PSD, $\phi^{P S D}$, was selected according to Eqn. (3) in such a way that the whole intensity of the modulation spectrum appeared only in one of the two relevant orthogonal modulation spectra. This spectrum served later as a reference modulation spectrum for $\mathrm{c}-\mathrm{ME}$ of HSA by cholate. Experimental conditions: All spectra were measured with parallel polarized light on a Ge MIRE; $\mathrm{T}=25^{\circ} \mathrm{C}$; angle of incidence $\Theta=45^{\circ}$; number of active internal reflections $\mathrm{N}$ $=19.6 \pm 1$. Buffer: $10 \mathrm{mM} \mathrm{Na} \mathrm{HPO}_{4} / \mathrm{NaH}_{2} \mathrm{PO}_{4} \mathrm{pH}$ $7.2,150 \mathrm{mM} \mathrm{NaCl}, 0.02 \%$ (w/v) sodium azide, modulation period: $9.6 \mathrm{~min}$; flow rate: $1 \mathrm{ml} / \mathrm{min}$.

Fig. 10. Cholate concentration-modulated excitation (c-ME) of an immobilized monolayer of human serum albumin (HSA). For the sake of comparison, Fig. $9 \mathrm{c}$ is shown again as (a) because it serves as reference modulation spectrum for c-ME of HSA by cholate. (a) and (b) are phase resolved spectra calculated with the same $\Phi^{P S D}$ that maximised the amplitudes of the modulated response according to Eqn. (3). (c) is the difference between (b) and (a). It reflects cholate bound to HSA and structural responses of HSA upon interaction. This spectrum is shown with expanded scale in the amide I and II region and both polarizations in Fig. 11. Experimental conditions: As in Fig. 9.

Concerning the resulting modulation spectra, attention should be drawn to the following facts: (i) the orthogonal spectra $\left(\varphi\right.$ and $\left.\varphi+90^{\circ}\right)$ of both the fundamental frequency $(\omega)$ and the first harmonic $(2 \omega)$ are not similar, i.e. cannot be converted into each other by a simple multiplication with a factor. This can happen only if different absorbing components have different relaxation times leading to different phase lags in the response of the PLL to T-ME. As a consequence the stimulated process must have accessible relaxation times comparable to the stimulation period. (ii) The existence of a $2 \omega$ response 


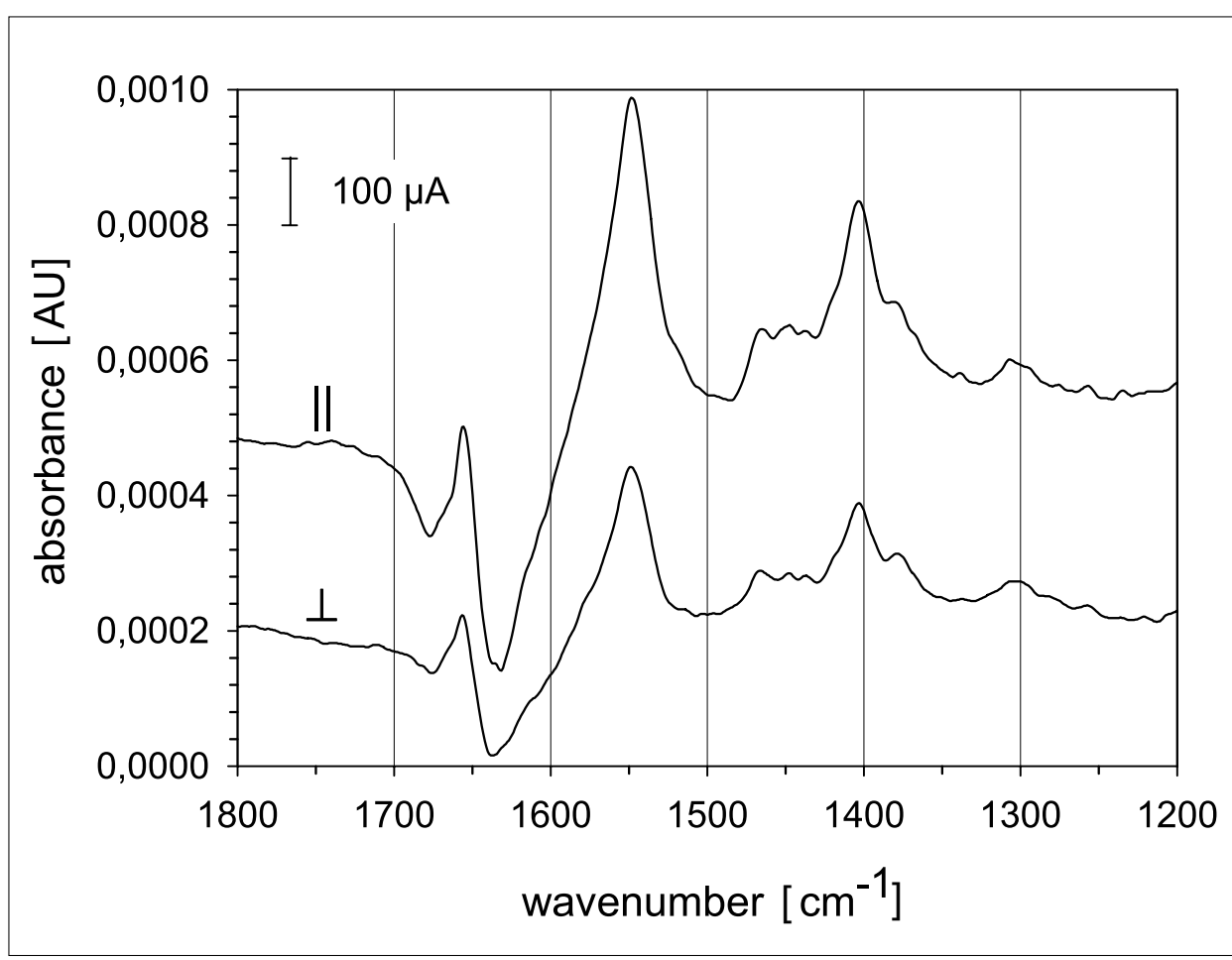

Fig. 11. Cholate concentration-modulated excitation (c-ME) of an immobilized monolayer of human serum albumin (HSA). Difference modulation spectra according to Fig. $10 \mathrm{c}$ measured with parallel (II) and perpendicular $(\perp)$ polarized incident light. The spectra are dominated by the asymmetric and symmetric carboxylate stretching vibrations of bound cholate, $v_{\mathrm{as}}\left(\mathrm{COO}^{-}\right)$at $1543 \mathrm{~cm}^{-1}$ and $v_{\mathrm{s}}\left(\mathrm{COO}^{-}\right)$ at $1405 \mathrm{~cm}^{-1}$, respectively. From the integrated intensity of the latter a surface concentration of bound cholate was calculated to be $(4.67 \pm 0.82) \cdot 10^{-11} \mathrm{~mol} / \mathrm{cm}^{2}$, using a integrated molar absorption coefficient of $(1.3 \pm 0.2) \cdot 10^{7} \mathrm{~cm} / \mathrm{mol}$ (range of integration: $1349 \mathrm{~cm}^{-1}-1485 \mathrm{~cm}^{-1}$ ). This corresponds to $8.4 \pm 0.6$ cholate molecules per HSA at a bulk cholate concentration of $10 \mathrm{mM}$. In addition a narrow band at $1656 \mathrm{~cm}^{-1}$, i.e. in the amide I region, appears synchronously with cholate binding to HSA. A tentative interpretation is increase of $\alpha$-helical structures upon cholate binding. Experimental conditions: As in Fig. 9.

of the sample in spite of the absence of such a component in the temperature stimulation gives unambiguous evidence of a non-linear kinetic process (2nd order or more complex).

By calculating a series of phase-resolved spectra with phase angles, we were able to select phase-resolved spectra where the contribution of components with high concentrations ( $\alpha$-helix, $\beta$-pleated sheet) was reduced to a minimum (see Eqn. (3)). From those spectra it was possible to detect and evaluate the properties of weak absorption bands featuring phase lags different from the selected $\phi^{P S D}$. These bands result generally from transients appearing during the folding process, because their phase lags are different from the phase lags of the initial and final structure. Although those transients have only small concentrations some of them could be detected by ME-spectroscopy [6]. Moreover, recent frequency dependent measurements revealed insight into kinetics and reaction scheme of the reversible conversion between $\alpha$-helix and $\beta$-sheet (to be published).

This example demonstrates the capability of ME-spectroscopy for experimentally separating overlapping absorption bands, and additionally giving access to kinetic properties of the stimulated system.

\section{Conclusions and Perspectives}

Without claiming completeness, we have reported in this article on some FTIR ATR applications requiring enhanced sensitivity and long term baseline stability. We did not report $e . g$. on a variety of structural studies with polymers [28-30] and on the interaction of pesticides with model lipid membranes [31], as well as on the basic preparation of model membranes for ATR studies [32]. Such model membranes have turned out to be also adequate for the study of interaction with pharmaceuticals such as local anesthetics [33][34], peptide antibiotics [35][36] and hormones [37][38].

A promising new field of application of our techniques is found in biosensor and chemical sensor development. Such surface-attached assemblies often consist of several steps of surface chemical synthesis [39]. This article reports on the development of a chemical sensor for the detection of serine-proteases on planar optical waveguides. We plan to perform organic synthesis building up specific molecular assemblies at the surface a GE MIRE. The novelty compared to earlier work is that synthesis will be performed directly in

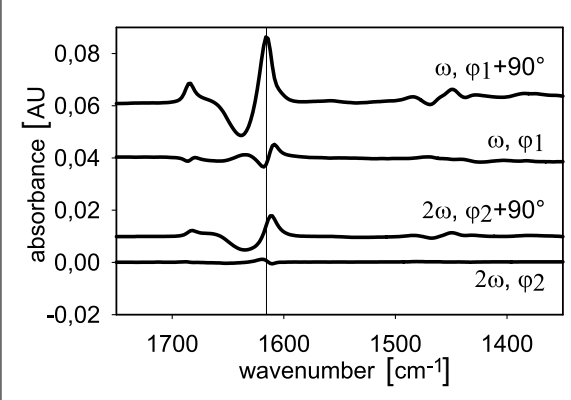

Fig. 12. Temperature modulated excitation (T$M E)$ of a thin $\mathrm{D}_{2} \mathrm{O}$ hydrated layer of poly-L-lysine (PLL) on a Ge MIRE. Orthogonal phase-resolved spectra of PLL as evaluated by PSD in the fundamental $(\omega)$, and first harmonic frequency $(2 \omega)$. Note that the orthogonal spectra obtained with both demodulation frequencies are not similar, which gives unambiguous evidence for phase resolution, i.e. access to time resolution of stimulated processes in PLL. The presence of a response in the first harmonic $(2 \omega)$, in spite of pure sinusoidal T-ME gives evidence of non-linear kinetic steps in the course of the reversible $\alpha$ helix to antiparallel $\beta$-pleated sheet conversion. Comparison of all presented modulation spectra in this figure show unambiguously that the most intense band of $\beta$-pleated sheet consists of two overlapping components at 1609 and $1615 \mathrm{~cm}^{-1}$, which could be experimentally resolved due to a slight difference in the phase lag with respect to the stimulation. Experimental conditions: Ge MIRE; angle of incidence $\Theta=45^{\circ}$; relative humidity $\approx 80 \% \mathrm{D}_{2} \mathrm{O}$; temperature $\mathrm{T}=26 \pm 2{ }^{\circ} \mathrm{C}$ modulation period $60 \mathrm{~s}$.

the SBSR flow-through cell (Fig. 1d) in the spectrometer, resulting in permanent monitoring of the state of the reactions by SBSR FTIR ATR technique or by concentration modulated excitation (c-ME). In the latter case the dissolved chemicals will be periodically pumped through and washed out of the sample cuvette, leading to a stepwise progression of the synthesis, which can be monitored on a molecular level with the validated high sensitivity and baseline stability provided by modulation spectroscopy. Having a permanent information on the state of the reaction at the surface will enable the operator to optimize relevant parameters like reaction time, temperature, concentration of reactants etc. in a very short time. It should be noted, however, that the advantage of special FTIR ATR techniques over other methods is only substantiated in the state of development of new surface chemical procedures. Once a synthetic procedure is optimized, optical waveguide and surface plasmon resonance [40] techniques will be significantly less time-consuming than FTIR ATR.

Received: February 8, 2005 
[1] U.P. Fringeli, D. Baurecht, T. Bürgi, M. Siam, G. Reiter, M. Schwarzott, P. Brüesch, in 'Handbook of Thin Film Materials, Vol.2', Ed. H.S. Nalwa, Academic Press, San Diego, 2001, p. 191.

[2] Special ATR equipment was obtained from OPTISPEC, Rigistrasse 5, CH-8173 Neerach (Switzerland). http://www.optispec.ch

[3] U.P. Fringeli, in 'Biologically Active Molecules', Ed. U.P. Schlunegger, Springer Verlag, Berlin, 1989, p. 241.

[4] U.P. Fringeli, H.H. Günthard, D. Baurecht, in 'Infrared and Raman Spectroscopy of Biological Materials', Ed. H.U. Gremlich, B. Yan, Marcel Dekker, New York, 2000, p. 143.

[5] D. Baurecht, U.P. Fringeli, Rev. Sci. Instrum. 2001, 72, 3782.

[6] M. Müller, R. Buchet, U.P. Fringeli, J. Phys. Chem. 1996, 100, 10810.

[7] D. Baurecht, I. Porth, U.P. Fringeli, Vibrational Spectroscopy 2002, 30, 85.

[8] M. Schwarzott, P. Lasch, D. Baurecht, D. Naumann, U.P. Fringeli, Biophys. J. 2004, 86, 285.

[9] B. Desbat, M. Reylafon, T. Buffeteau, C. Destrade, J.J. Bonvent, H.T. Nguyen, Ferroelectrics 1992, 129, 43.

[10] R. Wirz, T. Burgi, W. Lindner, A. Baiker, Anal. Chem. 2004, 76, 5319.

[11] A. Gisler, T. Bürgi, A. Baiker, J. Catal. 2004, 222, 461.

[12] T. Bürgi Chimia 2003, 57, 623.

[13] A. Gisler, T. Burgi, A. Baiker, Phys. Chem. Chem. Phys. 2003, 5, 3539.

[14] R. Wirz, T. Bürgi, A. Baiker, Langmuir 2003, 19, 785.
[15] T. Burgi, A. Baiker, J. Phys. Chem. B 2002, 106, 10649.

[16] T Q. Wu, X.S. Lu, S.Z.Yang, B.H. Zhang, Spectroscopy and Spectral Analysis, 2002, $22,25$.

[17] I. Noda I, G.M. Story, C. Marcott, Vibrational Spectroscopy 1999, 19, 461.

[18] M. Forster, K. Loth, M. Andrist, U.P. Fringeli, H.H. Günthard, Chem. Phys. 1976, $17,59$.

[19] H.H. Günthard, Ber. Bunsenges. Phys. Chem. 1974, 78, 1110.

[20] G. Reiter, M. Siam, D. Falkenhagen, W. Gollneritsch, D. Baurecht, U.P. Fringeli, Langmuir 2002, 18, 5761.

[21] G. Reiter, N. Hassler, V. Weber, D. Falkenhagen, U.P. Fringeli, BBA-Proteins and Proteomics 2004, 1699, 253.

[22] M. Schwarzott, H. Engelhardt, T. Klühspies, D. Baurecht, D. Naumann, U.P. Fringeli, Langmuir 2003, 19, 7451.

[23] A. Paul, H. Engelhardt, U. Jakubowski, W. Baumeister, Biophys. J. 1992, 61, 172.

[24] D.C. Carter, J.X. Ho, Adv. Protein Chem. 1994, 45, 153.

[25] A. Roda, G. Cappelleri, R. Aldini, E. Roda, L. Barbara, J. Lipid Res. 1982, 23, 490.

[26] D. Baurecht, W. Neuhäusser, and U.P. Fringeli, in 'Proceedings of the 11th International Conference on Fourier Transform Spectroscopy', Ed. J.A. de Haseth, The American Institute of Physics, Woodbury, New York, 1998, p. 367.

[27] T. Peters, Jr., 'All about Albumin', Academic Press, San Diego, 1995.

[28] M. Müller, T. Rieser, K. Lunkwitz, J. Meier-Haack, Macromol. Rapid Commun. 1999, 20, 607 .
[29] M. Müller, in 'Handbook of Polyelectrolytes and Their Applications', Eds. S.K. Tripathy, J. Kumar, H.S. Nalwa, American Scientific Publishers, New York, 2002, 14, 1.

[30] M. Müller, B. Kessler, K. Lunkwitz, J. Phys. Chem. B 2003, 107, 8189.

[31] M. Siam, G. Reiter, R. Hunziker, B. Escher, A. Karpfen, A. Simperler, D. Baurecht, U.P. Fringeli, BBA-Biomembranes 2004, 1664, 88.

[32] P. Wenzl, M. Fringeli, J. Goette, U.P. Fringeli, Langmuir 1994, 10, 4235.

[33] M. Schöpflin, U.P. Fringeli, X. Perlia, J. Am. Chem. Soc. 1987, 109, 2375.

[34] U.P. Fringeli, Chimia 1992, 46, 200.

[35] U.P. Fringeli, M. Fringeli, Proc. Natl. Acad. Sci. USA 1979, 76, 3852.

[36] U.P. Fringeli, J. Membrane Biol. 1980, 54, 2103.

[37] H.U. Gremlich, U.P. Fringeli, R. Schwyzer, Biochemistry 1983, 22, 4257.

[38] H.U. Gremlich, U.P. Fringeli, R. Schwyzer, Biochemistry 1984, 23, 1808.

[39] G. Zhylyak, V. Ramoz-Perez, M. Linnhoff, T. Hug, D. Citterio, U.E. Spichiger-Keller, 'Detection of Serine-Proteases on Planar Optical Waveguides' Optics and Lasers in Engineering, Elsevier Science Ltd. (Amsterdam), 2004, 43/3-5, 603.

[40] Biacore International AB, Head Office, Puits-Godet 12, CH-2000 Neuchâtel, (Switzerland). http://www.biacore.com/ k2/literature/core.lasso 